\title{
Gallstones and Bariatric Surgery: To Treat or Not to Treat?
}

\author{
Samir Johna ${ }^{1}$
}

Published online: 26 July 2016

(c) Société Internationale de Chirurgie 2016

The relationship between the gall bladder and morbid obesity, and the strategy used by surgeons for its management can be passive-aggressive. Morbidly obese patients are more likely to develop cholelithiasis. On the other hand, bariatric surgery predisposes to gall bladder stone formation and potentially symptomatic cholelithiasis. The indication for cholecystectomy and its timing in relationship to bariatric surgery is a moving target. In this editorial, we review the current practice and what the literature has to say about it, particularly, what to do with the normal gall bladder and the asymptomatic cholelithiasis in anticipation of bariatric surgery. It seems that, based on the work done by Morais et al. [1], in this issue of the WJS, removing normal gall bladders or performing cholecystectomy for asymptomatic cholelithiasis is not justified when a bariatric procedure is done, a deep sigh of relief for bariatric surgeons. However, the evidence is hardly conclusive, and the debate will continue until prospective randomized trials are carried out. To close the loop on this surgical dilemma, we need to study the proper timing of intervention for one procedure in relationship to the other. Until then, the jury is out.

Are you tired of politics and the 2016 political rallies of the US presidential race? Do you want to consider some other controvery for a change, like surgery? Well, do not hold your breath! Politics is everywhere. Voting for the right option in the management of gall bladder disease if you are contemplating a bariatric procedure is no

Samir Johna

samir.johna@gmail.com

1 Loma Linda University School of Medicine, Loma Linda, CA, USA exception. Should you be a liberal or a conservative? That is the question.

In an attempt to answer this question, experts in bariatric surgery tried several options, ranging from one extreme to another. They relied on the limited available data sandwiched in space between expert opinions. Many issues of concern, some technical and other financial, dictated this indecision. Most bariatric surgeons, at least in the USA, leave the management of the gall bladder disease to general surgeons unless a decision is made to remove the gall bladder concomitantly. The general surgeons are faced with the technical difficulties of removing the gall bladder in the morbidly obese patient if cholecystectomy has to be done first. Having to go through a second (bariatric) procedure later is another concern for the patient. On the other hand, the bariatric surgeons do not want to add more variables that may complicate their outcomes if a concomitant procedure is to be done. Removing the gall bladder during a bariatric surgery prolongs the operative time and may require additional ports for optimal access. Data have even shown a higher morbidity and longer hospital stay if both procedures are combined [2]. Equally important, concomitant cholecystectomy is not reimbursed. On the hand, if the gall bladder acts up after the bariatric procedure, the general surgeon may have the advantage of operating when the extra weight is off, but is faced with possible lysis of adhesions, particularly, when the bariatric procedure was done open.

We know that gall bladder disease is associated with morbid obesity [3]. The addition of a bariatric procedure complicates the matter even further. Rapid weight loss and its metabolic derangements add additional burden to the biliary system [4], leading to a higher incidence and prevalence of gallbladder disease. Every patient offered a bariatric procedure falls in one of the following categories 
at baseline; normal gall bladder, asymptomatic cholelithiasis, and symptomatic cholelithiasis. After the procedure, patients with a normal in situ gall bladder may develop asymptomatic or symptomatic cholelithiasis. Patients with asymptomatic cholelithiasis at baseline may become symptomatic after the bariatric procedure, while the ones who were symptomatic yet did not have concomitant cholecystectomies will continue to suffer.

An additional problem involves the loss of access to the biliary tree after any form of gastric bypass [5]. In patients with cholelithiasis, are we risking migration into the bile duct while waiting for symptoms to develop? How hard is it for a skilled bariatric surgeon to remove a gall bladder (especially the asymptomatic one) during a sleeve or bypass? It might be argued that this kind of overthinking on the simple issue of cholecystectomy (the commonest procedure) may lead to more patient suffering. Given all of these possibilities, it is not hard to imagine why it is so difficult to reach a consensus.

A common approach today is to prescribe prophylactic ursodeoxycholic acid for the first 6 months after surgery during the period when the maximal weight loss is expected [6]. Most bariatric surgeons would leave the normal gall bladders and those with asymptomatic cholelithiasis in situ. Some bariatric surgeons refer the symptomatic patients to general surgeons for cholecystectomy before bariatric surgery is considered. A few bariatric surgeons will accept performing a concomitant procedure. So, is this approach supported by the current literature?

In this issue of the World Journal of Surgery, Morais et al. [1] addressed one aspect of the bigger question, "How should we manage the normal gall bladder and asymptomatic cholelithiasis in anticipation of a bariatric procedure? What are the risk factors for developing symptomatic cholelithiasis after a bariatric procedure?"

The authors prospectively followed 653 patients undergoing bariatric surgery. Three procedures were offered based on the patients' preoperative merits; RYGBP, gastric band, and sleeve gastrectomy. All patients had their gall bladder left in situ. In total, $12 \%$ of the followed patients had asymptomatic cholelithiasis at baseline, and the remainder had normal gall bladders. Patients were followed every 6 months for 3 years. Only $3.3 \%$ of the patients developed symptomatic cholelithiasis, of which only one third progressed to complicated gall bladder disease. Of interest, none of patients who developed symptomatic cholelithiasis had gall bladder stones at baseline. The authors concluded that removing normal gall bladders or performing cholecystectomy for asymptomatic cholelithiasis is not justified. Their data also showed higher chance of developing symptomatic disease if the patient had longer duration of obesity, a higher excess weight loss, and lower blood insulin levels. No statistically significant relationship was found between the type of the procedure performed and the development of gall bladder disease.

There are several limitations in this study. First, it is an observational study. Its retrospective nature is associated with inherent biases. Second, three different procedures were offered, each having a potentially different effect on the gall bladder. Finally, the allocation of patients to the type of the procedure was left to the discretion of the operating bariatric surgeon. Nevertheless, the authors provide in this study some convincing evidence that normal gallbladders and those with asymptomatic cholelithiasis can be left alone, a deep sigh of relief for bariatric surgeons. However, this evidence is far from being conclusive, and the debate will continue until prospective randomized trials are carried out. To close the loop on this surgical dilemma, we need to study the proper timing of intervention for one procedure in relationship to the other. Until then, the jury is out.

\section{References}

1. Morais M, Faria G, Preto J et al (2016) Gallstones and bariatric surgery: to treat or not to treat? World J Surg. doi:10.1007/s00268016-3639-2

2. Shiffman ML, Sugerman HJ, Kellum JM, Brewer WH, Moore EW (1991) Gallstone formation after rapid weight loss: a prospective study in patients undergoing gastric bypass surgery for treatment of morbid obesity. Am J Gastroenterol 86(8):1000-1005

3. Amaral JF, Thompson WR (1985) Gallbladder disease in the morbidly obese. Am J Surg 149(4):551-557

4. Ming-Li VK, Pulido N, Fajnwaks P, Szomstein S, Rosenthal R (2009) Predictors of gallstone formation after bariatric surgery: a multivariate analysis of risk factors comparing gastric bypass, gastric banding, and sleeve gastrectomy. Surg Endosc 23(7):1640-1644

5. Saget A, Facchiano E, Bosset PO et al (2010) Temporary restoration of digestive continuity after laparoscopic gastric bypass to allow endoscopic sphincterotomy and retrograde exploration of the biliary tract. Obes Surg 20(6):791-795

6. Sugerman HJ, Brewer WH, Shiffman ML et al (1995) A multicenter, placebo-controlled, randomized, double-blind, prospective trial of prophylactic ursodiol for the prevention of gallstone formation following gastric-bypass-induced rapid weight loss. Am J Surg 169(1):91-97 